\title{
Organisation and regulation of the cytoskeleton in plant programmed cell death
}

\author{
A Smertenko ${ }^{1}$ and VE Franklin-Tong, ${ }^{* 2}$
}

Programmed cell death $(\mathrm{PCD})$ involves precise integration of cellular responses to extracellular and intracellular signals during both stress and development. In recent years much progress in our understanding of the components involved in PCD in plants has been made. Signalling to PCD results in major reorganisation of cellular components. The plant cytoskeleton is known to play a major role in cellular organisation, and reorganization and alterations in its dynamics is a well known consequence of signalling. There are considerable data that the plant cytoskeleton is reorganised in response to PCD, with remodelling of both microtubules and microfilaments taking place. In the majority of cases, the microtubule network depolymerises, whereas remodelling of microfilaments can follow two scenarios, either being depolymerised and then forming stable foci, or forming distinct bundles and then depolymerising. Evidence is accumulating that demonstrate that these cytoskeletal alterations are not just a consequence of signals mediating PCD, but that they also may have an active role in the initiation and regulation of PCD. Here we review key data from higher plant model systems on the roles of the actin filaments and microtubules during PCD and discuss proteins potentially implicated in regulating these alterations.

Cell Death and Differentiation (2011) 18, 1263-1270; doi:10.1038/cdd.2011.39; published online 13 May 2011

The plant cytoskeleton comprises two dynamic interconnected arrays: microtubules and microfilaments. Microtubules polymerise from $\alpha-/ \beta$-tubulin heterodimers and microfilaments polymerise from actin monomers. It has recently been shown that the density of filamentous actin (F-actin) network undergoes periodic oscillations of polymerisation and depolymerisation, ${ }^{1}$ and alterations of this periodicity may act in perceiving signals and generating responses. Alterations to the actin cytoskeleton are regulated by actin-binding proteins (ABPs) that stimulate formation of new filaments, promote filament elongation, bundle filaments together, strengthen interaction between actin/tubulin subunits, induce severing and depolymerisation. ${ }^{2}$ Plant microtubules undergo dynamic instability ${ }^{3}$ under the control of microtubule-associated proteins (MAPs; reviewed by Hamada ${ }^{4}$ and Sedbrook ${ }^{5}$ ). The dynamic nature of F-actin and microtubules enables flexibility of cytoskeletal organisation, making it an integral part of signalling networks, translating developmental and environmental cues into cellular responses. ${ }^{6}$ Alterations to the cytoskeleton during programmed cell death (PCD) have been described in a variety of different plant systems (Table 1) and application of anti-cytoskeletal drugs implicate these alterations in playing an active a role in signalling to or mediating $\mathrm{PCD}$, rather than being merely a consequence of cellular changes induced by PCD. Here we review key data relating to this topic.

\section{F-actin and Microtubules Reorganise during Developmental PCD}

Developmental PCD is an integral part of tissue differentiation during normal growth (see other articles in this issue). In some cases it may take weeks (leaf senescence), days (embryo suspensor cells), or hours (in vitro differentiation of Zinnia tracheary elements). Tracheary elements are the main structural element of xylem and essential for water and nutrient conductance, and mechanical strength. Differentiation of tracheary elements requires localised deposition of oligosaccharide material (secondary cell wall). When deposition is complete, the contents of the cell are dismantled by PCD. ${ }^{7,8}$ In the case of Zinnia model system, before tracheary elements differentiate, cortical microtubules are oriented transversely and spaced randomly. ${ }^{9}$ Upon differentiation, microtubules form thick bundles between cytoplasmic domains containing virtually no microtubules (Table 1). Localised deposition of the secondary cell wall material is associated with microtubules. ${ }^{10}$ F-actin is required for the

\footnotetext{
${ }^{1}$ School of Biological and Biomedical Sciences, Durham University, Durham, DH1 3LE, UK and ${ }^{2}$ School of Biosciences, College of Life and Environmental Sciences, University of Birmingham, Birmingham B15 2TT, UK

${ }^{*}$ Corresponding author: VE Franklin-Tong, School of Biosciences, College of Life and Environmental Sciences, University of Birmingham, Edgbaston, Birmingham B15 2TT, UK. Tel: + 44121414 3702; Fax: + 441214141 5925; E-mail: v.e.franklin_tong @bham.ac.uk

Keywords: programmed cell death; cytoskeleton; microtubules; F-actin

Abbreviations: $A B P$, actin-binding protein; ADF, actin-depolymerising factor; CAP, cyclase-associated protein; F-actin, filamentous actin; HR, hypersensitive response; MAP, microtubule-associated protein; $\mathrm{PCD}$, programmed cell death; SI, self-incompatibility

Received 21.2.11; revised 15.3.11; accepted 16.3.11; Edited by P Bozhkov; published online 13.5.11
} 
Table 1 The fate of the cytoskeleton during plant PCD

\begin{tabular}{|c|c|c|c|}
\hline Process & Microtubules & F-actin & References \\
\hline \multicolumn{4}{|l|}{ Development } \\
\hline Tracheary elements differentiation & $\begin{array}{l}\text { Switch to transverse orientation } \\
\text { and then depolymerise }\end{array}$ & $\begin{array}{l}\text { Switch to transverse orientation and then } \\
\text { disassemble }\end{array}$ & $7-10$ \\
\hline Embryo development & $\begin{array}{l}\text { Switch to transverse orientation, } \\
\text { then depolymerise }\end{array}$ & Longitudinal thick long fibres & 12,13 \\
\hline \multicolumn{4}{|l|}{ Senescence } \\
\hline Arabidopsis leaves & Depolymerisation & & 16 \\
\hline \multicolumn{4}{|l|}{ Self-incompatibility response } \\
\hline $\begin{array}{l}\text { Self-incompatibility response in } \\
\text { incompatible pollen (Papaver rhoeas) }\end{array}$ & Depolymerisation & $\begin{array}{l}\text { Depolymerisation followed by } \\
\text { polymerisation and aggregation into } \\
\text { highly stable punctate foci }\end{array}$ & $27-31$ \\
\hline $\begin{array}{l}\text { Self-incompatibility response in } \\
\text { incompatible pollen (Pyrus pyrifolia) }\end{array}$ & & $\begin{array}{l}\text { Depolymerisation followed by } \\
\text { subsequent aggregation into punctate } \\
\text { foci }\end{array}$ & 33 \\
\hline \multicolumn{4}{|l|}{ Hypersensitive response } \\
\hline Linum usitatissimum/Melapsora lini & $\begin{array}{l}\text { Concentrate at the infection site, } \\
\text { disassembly after penetration }\end{array}$ & $\begin{array}{l}\text { Concentrate at the infection side, } \\
\text { disassembly after penetration }\end{array}$ & 42 \\
\hline Vigna unguiculata/Uromyces vignae & Depolymerisation & Concentration beneath the appressorium & 39 \\
\hline Glycine max/Phytophthora sojae & Depolymerisation & & 44 \\
\hline $\begin{array}{l}\text { Arabidopsis thaliana/Peronospora } \\
\text { parasitica }\end{array}$ & $\begin{array}{l}\text { Depolymerisation at the infection } \\
\text { site }\end{array}$ & $\begin{array}{l}\text { Formation of bundles directed toward } \\
\text { infection site }\end{array}$ & 40 \\
\hline Arabidopsis thaliana/Blumeria graminis & Partial depolymerisation & $\begin{array}{l}\text { Concentration beneath the appressorium, } \\
\text { then depolymerisation }\end{array}$ & 43 \\
\hline \multicolumn{4}{|l|}{ Treatment with fungal toxins } \\
\hline N. tabacum BY-2 cells/cryptogein & Depolymerisation & Bundling & 41,45 \\
\hline $\begin{array}{l}\text { A. thaliana leaves/Verticillium dahliae } \\
\text { toxins }\end{array}$ & Depolymerisation & & 66 \\
\hline
\end{tabular}

orientation of microtubules, ${ }^{9}$ and organisation of F-actin changes during the secondary cell wall deposition from a longitudinal network to transverse bundles located between the bundles of microtubules (Table 1). On completion of the secondary wall thickening, microfilaments and microtubules fragment, and the tonoplast ruptures. It is not known if the cytoskeleton has a role in processes immediately preceding tonoplast rupture or if its disintegration is required for the successful execution of PCD.

Somatic embryos of Norway spruce (Picea abies) develop in vitro from proliferating clusters of cells called proembryogenic masses. ${ }^{11}$ Early in development, spruce embryos establish an apical-basal pattern via specification of three structural components: an embryonal mass composed of proliferating meristematic spherical cells with dense cytoplasm, elongated embryonal tube cells, and giant vacuolated embryo suspensor cells. Embryonal tube cells are terminally differentiated and committed to death; they originate through re-iterated asymmetric divisions of stem-like cells of the embryonal mass cells and give rise to the suspensor cells. Suspensor cells undergo vacuolar PCD and dissociate at the distal part of the suspensor. ${ }^{11}$ Embryonal mass size increases until the completion of the embryogenesis and the embryonal tube and suspensor cells diminish until none are left in the mature embryos. Each cell-type exhibits different cytoskeletal organisation. Proliferating embryonal mass cells have dense arrays of randomly oriented F-actin and microtubules. In anisotropically expanding embryonal tube cells, the microtubules form a transverse cortical array and microfilaments are oriented longitudinally. ${ }^{12,13}$ In the suspensor cells microtubules fragment and disassemble, but tubulin levels remain stable ${ }^{12}$ whereas longitudinal microfilaments bundle and form long cables, which disappear at the onset of DNA fragmentation (Figures 1a and b; Table 1). The reorganisation of F-actin in the suspensor cells is accompanied by upregulation of three actin genes. ${ }^{13}$

Senescence is important for plant organ replacement due to aging (e.g. leaves) or following the completion of the organ function (e.g. post-pollinated flowers). However, to date, studies of the cytoskeleton in senescence-induced PCD are limited. Proteomic and gene transcriptional analyses reveal downregulation of tubulin genes and a decrease of tubulin protein levels, and upregulation of actin gene transcription and an increase in actin protein level in senescing Arabidopsis tissue culture cells and Petunia corollas. $^{14,15}$ During natural and dark-induced senescence in Arabidopsis leaves, the cortical microtubule network in the epidermis and mesophyll cells gradually depolymerises (Table 1). ${ }^{16}$ This disassembly is tightly controlled, as neighbouring stomatal cells have an intact microtubule network.

\section{Self-incompatibility (SI) can Trigger PCD and Alterations to the Cytoskeleton}

$\mathrm{SI}$ is a genetically controlled system mediated by interaction between cognate pollen and pistil $S$-determinants to prevent self-fertilisation. ${ }^{17}$ In Papaver rhoeas L. (the field poppy), $S$-determinants are novel, highly polymorphic proteins, PrsS and PrpS. ${ }^{18,19}$ PrsS triggers a $\mathrm{Ca}^{2+}$-dependent signalling 

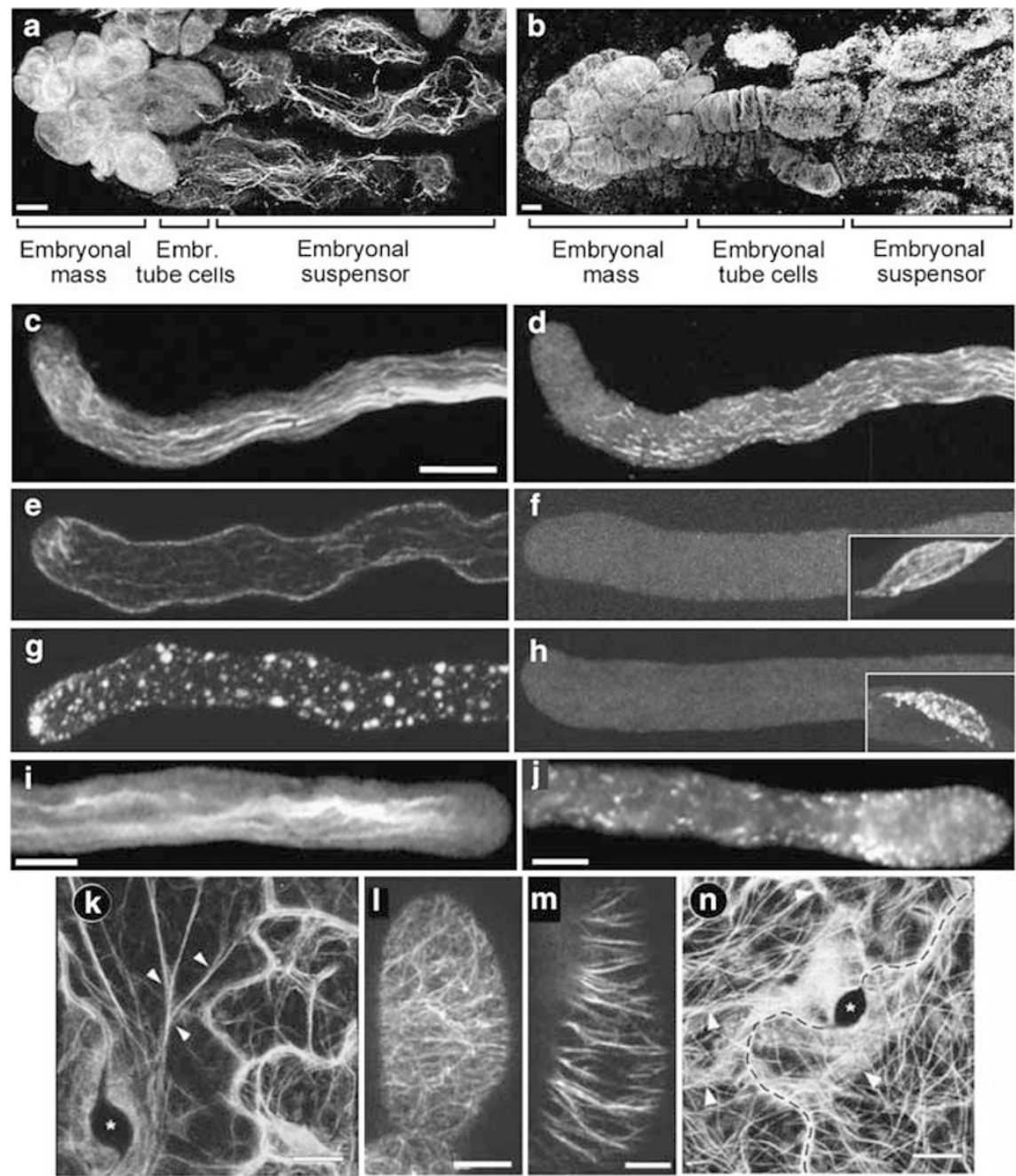

Figure 1 Organisation of F-actin and microtubules during PCD. (a and $\mathbf{b})$ Developmental PCD in Norway spruce somatic embryos triggers formation of longitudinal cables of actin (a) and fragmentation and depolymerisation of microtubules (b) in the embryo-suspensor cells. The sample was taken 7 days after withdrawal of growth factors. Scale bar $20 \mu \mathrm{m}$. (c-h) Papaver pollen tubes exhibit (c) distinctive actin microfilament arrays and (d) microtubule arrays. These are dramatically altered by the SI response. (e) F-actin bundles appear to depolymerise within $2 \mathrm{~min}$ and (f) most microtubules appear to be depolymerised within $1 \mathrm{~min}$, but the microtubule array around the generative nucleus (inset) is intact. (g) F-actin reorganises and aggregates into large 'punctate foci', but microtubules remain depolymerised (h). Scale bar $10 \mu \mathrm{m}$. (i and j) F-actin in Pyrus pyrifolia pollen tubes (i) forms punctate foci after SI-induction (j). Scale bar $10 \mu \mathrm{m}$. (k and $\mathbf{n}$ ) Hypersensitive response in $A$. thaliana attacked by virulent strain of $P$. parasitica. Asterisks indicate penetration sites and arrows indicate cytoplasmic strands. Microfilaments are organised in cables directed toward the infection site (k). Diffuse fluorescence, forming an apparent 'nebula' at the penetration site suggests microtubule depolymerisation (n). Scale bar $10 \mu \mathrm{m}$. (I and $\mathbf{m})$ Organisation of F-actin in control (I) and cryptogein-treated (m) tobacco BY-2 tissue culture cells. Scale bar $10 \mu \mathrm{m}$. (a, c, e, g, i and j) Staining with phalloidin derivatives conjugated to fluorescent probes; (b, d, $\mathbf{f}$ and $\mathbf{h})$ immunostaining with anti-tubulin antibody; (k) GFP-hTalin (actin-binding domain of Talin) fluorescence; (n) GFP-TUA6 (A. thaliana $\alpha$-tubulin 6) fluorescence; (I and $\mathbf{m}$ ) GFP-fABD2 (fimbrin actin-binding domain 2) fluorescence. (c, $\mathbf{d}, \mathbf{f}$ and $\mathbf{h}$ ) From ${ }^{31}$ Plant Physiology. (e and $\left.\mathbf{g}\right)$ From ${ }^{29}$ by kind permission from American Society of Plant Biologists. (i and $\left.\mathbf{j}\right)$ From ${ }^{33}$ by kind permission from Springer-Verlag Wien. ( $(\mathbf{k}$ and $\mathbf{n})$ From $^{40}$ by kind permission of John Wiley and Sons. (I and $\mathbf{m}$ ) From ${ }^{41}$ by kind permission from Oxford University Press

network leading to PCD in incompatible pollen. ${ }^{20-22}$ There is evidence that ROS, $\mathrm{NO}^{23}$ and an Sl-activated mitogenactivated protein kinase , $p 56^{24}$ signal to PCD. Evidence for PCD includes the appearance of cytochrome $c$ in the cytosol, dramatic morphological alterations to organelles ${ }^{25}$ activation of DEVDase, VEIDase and LEVDase, ${ }^{20,21}$ and DNA fragmentation; ${ }^{20,26}$ see Bosch and Franklin-Tong ${ }^{22}$ for a review. Growing pollen tubes contain a network of longitudinal F-actin bundles (Figure 1c) and microtubules (Figure 1d). SI triggers dramatic alterations to the cytoskeleton in incompatible pollen (Table 1). ${ }^{27-31}$ Within 1-2 min, most of F-actin bundles are depolymerised, but a population of cortical microfilaments remain (Figure 1e) and depolymerisation of cortical microtubules is observed, though microtubules around the generative cell are unaffected (Figure 1f). Next, F-actin forms stable 'punctate foci' (Figure 1g), but microtubules remain depolymerised and those microtubules surrounding the generative cell appear to degrade (Figure 1h).

Pyrus pyrifolia (pear) uses different pistil and pollen $S$-determinants (S-RNases and F-box proteins). However, there is evidence, including nuclear DNA fragmentation, a drop in mitochondrial membrane potential, and appearance of cytochrome $c$ in the cytosol that this SI system also triggers PCD in incompatible pollen tubes. ${ }^{32}$ Moreover, SI induction in vitro triggers reorganisation of the actin cytoskeleton in incompatible Pyrus pollen, resulting in the formation of 
punctate actin foci ${ }^{33}$ that appear very similar to those observed in Papaver SI (Figures 1i and j; Table 1). A more recent study suggests that ROS may be involved in mediating actin depolymerisation in Pyrus pollen tubes. ${ }^{34}$

\section{Involvement of the Cytoskeleton in the Hypersensitive Response (HR)}

When plants are attacked by pathogens, they mobilise defence reactions. This can use gene-for-gene resistance, involving interactions between host resistance $(R)$ genes and pathogen avirulence (Avr) genes, or non host-specific resistance $(\mathrm{NHR})$. Both interactions can trigger the $\mathrm{HR}$, a localised host cell death response around the pathogen infection site. HR can also be induced by elicitors produced by pathogens. Whether HR-PCD has a role in the inhibition of pathogen growth or resistance is unclear. However, there is extensive evidence for PCD being involved in $\mathrm{HR}^{35-38}$ (see related articles in this issue). Despite a wealth of information relating to the molecular genetics, cell biology, and biochemistry of host-pathogen interactions, relatively few studies have examined the role of the cytoskeleton in HR-PCD. It is well established that cytoskeletal reorganisation is triggered, and there is increasing evidence that the actin cytoskeleton can have an important role in HR-PCD.

During the early stages of infection, changes to F-actin organisation are usually apparent at the site of contact of the pathogen with the host plant. F-actin is consistently aggregated or bundled near the site of pathogen entry (Figure $1 \mathrm{k}){ }^{39,40}$ This type of reorganisation of F-actin has been observed as a plant response to attempted pathogen invasion in many interactions; see Table 1. Treatment of tobacco BY-2 cells with the elicitor, cryptogein, from Phytophtora cryptogea, also causes reorganisation and bundling of F-actin (Figures $1 \mathrm{l}$ and $\mathrm{m}$ ). ${ }^{41}$ Later stages of HR are often accompanied by depolymerisation of F-actin. ${ }^{42,43}$

Microtubule organisation is also affected by defence responses, but the response is variable. Disruption and apparent depolymerisation of the microtubule network at the penetration site has been observed in several systems (Figure $1 \mathrm{n}),{ }^{39,40,44}$ and it has been suggested that this might be an early sign of the HR. ${ }^{39,42}$ In other studies microtubules, like F-actin, concentrate at the infection site and undergo bundling, ${ }^{42}$ but some studies ${ }^{43}$ found no changes in the microtubule organisation upon pathogen attack. Treatment with cryptogein induces depolymerisation of microtubules ${ }^{41,45}$ providing further support of the idea that microtubules are involved in HR.

\section{Evidence that Actin Dynamics are Involved in Mediating PCD}

Use of a pharmacological approach has demonstrated that changes in actin dynamics can trigger PCD in animal, yeast, and plant cells. ${ }^{46}$ Experiments with toxins that interfere with actin and microtubule polymerisation dynamics provide important evidence that alterations to their dynamics can either trigger PCD or perturb PCD progression in plants (Figure 2). a
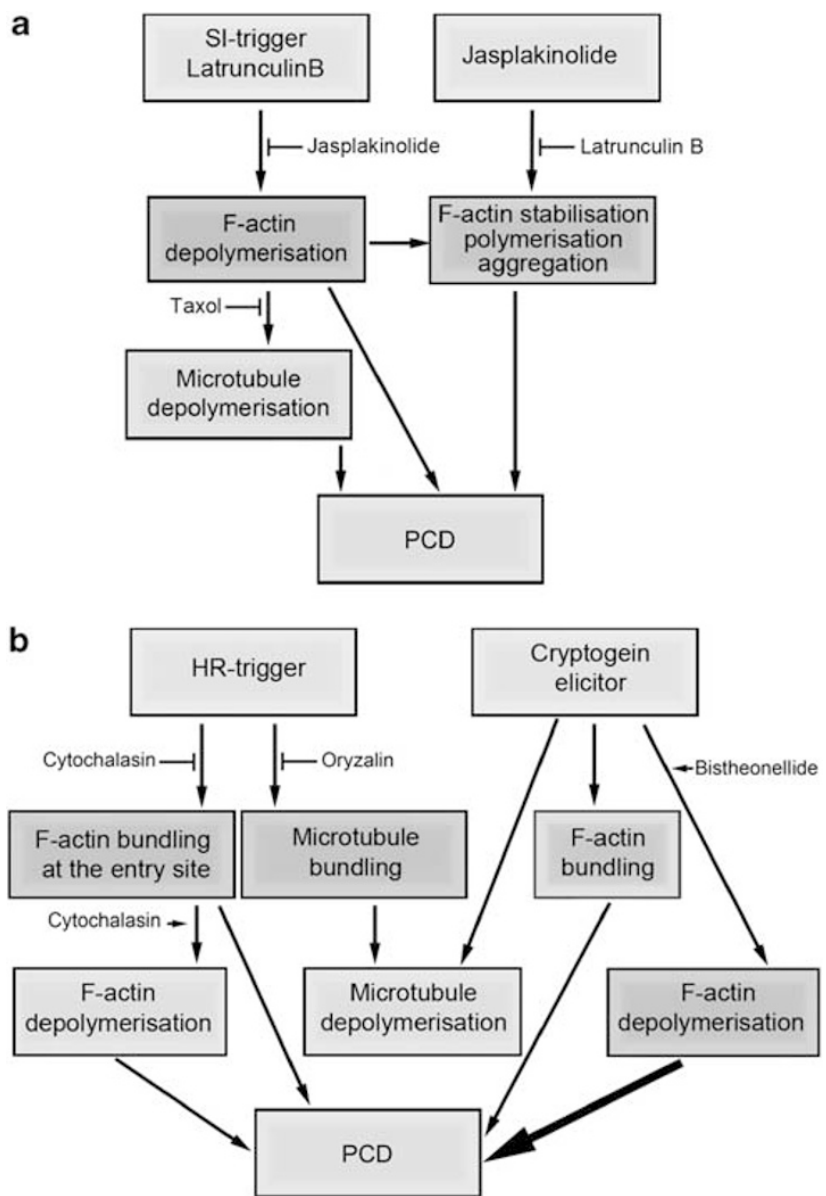

Figure 2 Analysis of the effect of microtubule and actin drugs on PCD. (a) SI response. SI in Papaver pollen triggers F-actin depolymerisation, then stabilisation, microtubule depolymerisation, and PCD. There is evidence using cytoskeletal drugs that both actin and microtubule cytoskeleton dynamics have a role in mediating PCD. Both the SI ligand PrsS and artificial depolymerisation of actin using Latrunculin B stimulates PCD in pollen. PCD can be alleviated by pre-treatment with jasplakinolide. Artificial depolymerisation of actin using Latrunculin B shows that this can trigger microtubule depolymerisation. Although artificial depolymerisation of microtubules using oryzalin does not stimulate PCD, this depolymerisation is important for the execution of PCD because treatment of pollen with the microtubule-stabilising drug taxol alleviates PCD. These data suggest that actin depolymerisation triggers microtubule depolymerisation and the two events together have a role in SI-mediated $P C D$, although actin depolymerisation (if great enough) alone is sufficient to trigger PCD. Treatment of pollen with Jasplakinolide also triggers PCD and this effect can be alleviated by the treatment by Latrunculin $\mathrm{B}$, suggesting that actin stabilisation (observed during $\mathrm{SI}$ ) also has a role in triggering PCD. However whether SI-mediated actin stabilisation itself triggers PCD has yet to be established. (b) HR. HR signals trigger bundling of F-actin and microtubule depolymerisation around the site of pathogen entry. Treatment with the F-actin depolymerising drug cytochalasin D and the microtubule depolymerising drug oryzalin can prevent PCD in some systems. F-actin depolymerises during later stages of $P C D$, and treatment with cytochalasin $D$ can promote PCD. There appears to be no evidence that microtubule bundling has a role in mediating PCD in HR as taxol has no effect on levels of HR-PCD. The possible crosstalk between microtubules and F-actin has not yet been characterised. Treatment with the elicitor, cryptogein, induces depolymerisation of microtubules and bundling of F-actin. F-actin bundling may regulate the pace of PCD, as treatment with the actindepolymerising drug, bistheonellide, speeds up PCD

Treatment of early Picea embryos with the actin-depolymerising drugs, Latrunculin $B$ and cytochalasin $D$, increases the rate of cell death, and inhibits differentiation of the 
suspensor. ${ }^{12,13}$ This suggests that actin polymerisation is important for initiating or mediating relatively slow vacuolar PCD in the embryo suspensor. In contrast, treatment of differentiating tracheary elements with cytochalasin $B$ has no effect on PCD, but perturbs differentiation. ${ }^{9}$

In Papaver pollen, relatively transient, but substantial depolymerisation of $\mathrm{F}$-actin by Latrunculin $\mathrm{B}$ was sufficient to trigger PCD. Moreover, use of jasplakinolide, which stabilises actin filaments and stimulates polymerisation, to counteract the F-actin depolymerisation induced by $\mathrm{SI}$ or Latrunculin $B$, significantly reduces levels of $\mathrm{PCD}^{47}$ (Figure 2a). This implicates actin depolymerisation in mediating SI-induced PCD. Jasplakinolide itself can trigger PCD, ${ }^{47}$ suggesting stabilisation also can trigger PCD. As the Sl-induced actin foci are extremely stable, ${ }^{29}$ stabilisation may also have a role in mediating SI-PCD. As either actin stabilisation or depolymerisation can stimulate PCD, it has been proposed that the fine balance of actin polymerisation dynamics is important for a 'healthy' situation, and that sustained changes to actin dynamics in either direction have a functional role in initiating PCD. Recent data from a different SI system, Pyrus, using the drugs cytochalasin B, which prevents actin polymerisation, and phalloidin, which stabilises F-actin, provides further evidence that actin polymerisation status may be involved in mediating $\mathrm{PCD} .^{34}$

Direct evidence for a role for F-actin in HR-PCD is limited. Several studies have shown that cytochalasins affect resistance to pathogens, ${ }^{48}$ suggesting a role for actin polymerisation, but as resistance is not always accompanied by HR-PCD, we do not consider these studies here. More direct evidence for a role for the actin cytoskeleton in HR-PCD is provided by data showing that cytochalasins can suppress cell death triggered by Phytophthora infestans in potato; cytochalasin $D$ treatment also delayed induction of key defence markers and decreased the accumulation of $P R$ proteins, with concomitant alleviation of cell death in potato tubers treated with $P$. infestans cell wall extracts (Figure 2b). ${ }^{49,50}$ These data suggest that this HR-PCD requires $\mathrm{F}$-actin polymerisation.

In contrast, in tobacco, cytochalasins can induce HR defence responses in a dose-dependent manner. ${ }^{51}$ Moreover, cryptogein-induced PCD in tobacco tissue culture cells is accelerated in the presence of the actin-depolymerising drug bistheonellide (Figure 2b). ${ }^{41}$ This suggests that actin depolymerisation has a key role in the induction of HR-PCD. Cytochalasin D did not affect the induction of the HR in wildtype Arabidopsis when coinoculated with Pseudomonas syringae pv tomato, but in an Atadf4 mutant that normally suppresses HR mediated by $P$. syringae pv tomato, it allowed a high percentage of leaves to generate the HR. ${ }^{52}$ This strongly suggests a role for actin cytoskeleton alterations, mediated by AtADF4, being involved in defence responses. Together these studies provide strong evidence implicating a causal relationship between actin polymerisation status and HR-PCD (Figure 2b). It has been suggested that the dynamic state of F-actin may have a role in the HR response. ${ }^{53}$ Thus, defence signalling networks may utilise manipulation of F-actin dynamics to transduce the HR-PCD signal. However, exactly how actin status relates to HR-PCD and how alterations are achieved is, in many cases, as yet, unclear.

\section{Evidence that Microtubule Dynamics can influence PCD}

Although there are clear data that microtubules reorganise during PCD in plant cells, the role of microtubule dynamics in initiating PCD in plants is currently poorly understood. Use of the microtubule depolymerising drug, oryzalin, or the microtubule stabilising drug, taxol, revealed that neither microtubule depolymerisation nor stabilisation alone was sufficient to trigger PCD in Papaver pollen. However, preventing microtubule depolymerisation using taxol significantly reduced SIinduced DEVDase activity. ${ }^{31}$ Thus, although depolymerisation of tubulin alone is not sufficient to trigger PCD, it is required for proper progression of the SI-PCD pathway. $\mathrm{F}$-actin depolymerisation using Latrunculin B triggers microtubule depolymerisation, and stabilisation of F-actin following $\mathrm{SI}$ induction resulted in alleviated or delayed microtubule depolymerisation $^{31}$ (Figure 2a). This suggests that actin signals to microtubules and that the two networks may act in concert to mediate the progression of PCD in pollen.

Use of microtubule drugs to investigate the role of microtubules in HR-PCD has been limited. Experiments altering microtubule dynamics using taxol or oryzalin before infection had no effect on HR-PCD. ${ }^{39}$ Treatment with drugs, that depolymerise microtubules before they bundle, abrogates the differentiation of tracheary elements, whereas treatments after microtubule bundling affects the shape of tracheary elements, ${ }^{10}$ but no effects on PCD have been reported. The possibility that microtubules may act in concert with actin (as found in the SI system) has not been examined. More analysis is required before firm conclusions can be drawn on the role of microtubule dynamics in HR-PCD or developmental PCD.

\section{How is Regulation of the Cytoskeleton to Mediate PCD Achieved?}

ABPs regulate $\mathrm{F}$-actin polymerisation and organisation in plant cells ${ }^{2}$ and are likely to have a role in mediating PCD. To date rather few $A B P s$ have been examined in the context of PCD. Only actin-depolymerising factor (ADF) has been shown to have a role in the regulation of HR-PCD. ${ }^{48,52}$ ADF severs F-actin, promotes dissociation of monomeric actin from the pointed end and increases F-actin turnover, ${ }^{54}$ so is important for the reorganisation of actin filaments. Proteomic analysis of Petunia flowers reveals downregulation of a basic isoform and upregulation of an acidic isoform of $A D F,{ }^{15}$ suggesting ADF may be phosphorylated during senescence. As phosphorylation inhibits ADF activity, ${ }^{55}$ it may contribute to altered F-actin dynamics during PCD. The role of AtADF4 in mediating defence signalling through modification of the actin cytoskeleton has recently been demonstrated using an Atadf4 mutant. Data suggests that Arabidopsis AtADF4, which has activities expected of a typical ADF, is involved in regulating F-actin depolymerisation during HR-PCD. ${ }^{52}$ However, as plants have many ADF isotypes, there is much to learn still.

During SI-mediated PCD in Papaver pollen, the very rapid $\mathrm{F}$-actin depolymerisation that has been shown to be important and necessary for $P C D^{46}$ is thought to be mediated by profilin and PrABP80, a gelsolin-like protein with $\mathrm{Ca}^{2+}$-dependent severing activity. ${ }^{56}$ Soon afterwards, ADF associates with 
punctate actin foci. ${ }^{29} \mathrm{SI}$-induced cytosolic acidification during PCD initiation ${ }^{21}$ is likely to be responsible for this change in localisation and/or activity, as most ADFs have high F-actindepolymerising activity at alkaline $\mathrm{pHs}$, whereas at acidic $\mathrm{pHs}$, most ADFs preferentially bind to F-actin. Thus, acidification and consequent loss of ADF actin-severing activity may regulate the formation and stability of actin foci. Cyclaseassociated protein (CAP) also rapidly associates with SIinduced punctate actin foci. ${ }^{29}$ In yeast (S. cerevisiae), CAP/ Srv2p localises to actin patches and is required for the formation of F-actin aggregates ${ }^{57}$ and is found in the actin 'bodies' in stressed, quiescent yeast. ${ }^{58}$ As CAP/ Srv2p binds to adenylate cyclase and facilitates initiation of cAMP signalling during PCD initiation in yeast, ${ }^{59}$ it may mediate foci formation and may, perhaps, link actin reorganisation to PCDsignalling networks in plant cells too. Surprisingly, fimbrin, a major F-actin bundling and cross-linking protein did not associate with the SI-induced F-actin foci. ${ }^{29}$

Other proteins associated with the SI-induced actin foci, identified using mass spectrometry, include 14-3-3 proteins, a chaperonin subunit, TCP1, and heat-shock proteins. ${ }^{30}$ These proteins are implicated in interacting with the cytoskeleton during stress. For example, chaperonins have high affinity for denatured actin and tubulin. Heat shock proteins have been reported to cross-link and stabilise F-actin. Their identification provides clues to events involving actin alterations during PCD.

The limited available data suggest that the reorganisation of F-actin during PCD may involve several scenarios. Two possibilities are outlined in Figure $3 a$. The first is characterised by the formation of bundled actin cables, and has been reported in several examples of HR and developmental PCD. ${ }^{12,13,40,41,60-62}$ This is likely to involve reduced activity of severing and barbed end capping proteins, and activity of bundling proteins (Figure $3 \mathrm{a}$ ), though no candidates have been identified in these systems yet. In the second scenario, observed in the SI response, F-actin initially undergoes depolymerisation, followed by the formation of actin foci. A gelsolin-like protein (PrABP80) and profilin are candidate ABPs that may be responsible for mediating F-actin depolymerisation; CAP and ADF are likely to promote foci formation (Figure 3a).

Microtubules consistently depolymerise in many model systems undergoing PCD, including HR, SI response, and embryo suspensor. Microtubules attenuate F-actin dynamics by slowing polymerisation and depolymerisation rates. ${ }^{1}$ As depolymerisation of microtubules may allow more rapid restructuring of the F-actin network, careful analysis of the dynamic parameters of microtubules and microfilaments during PCD is essential to obtain insights into their contribution to PCD. Key changes in the microtubule dynamics during PCD and proteins implicated so far in these changes are summarised in Figure $3 \mathrm{~b}$.

Cortical microtubules in differentiated plant cells are predominantly bundled. ${ }^{63}$ The $25 \mathrm{~nm}$ spacing of microtubules in some bundles is attributed to MAPs, specifically, MAP65. ${ }^{64}$ Evidence that microtubule bundling may be important for the initiation of PCD is provided by the embryogenesis defective mutant of Picea that has no MAP65 associated with the cortical microtubules and does not undergo PCD. ${ }^{12}$ During

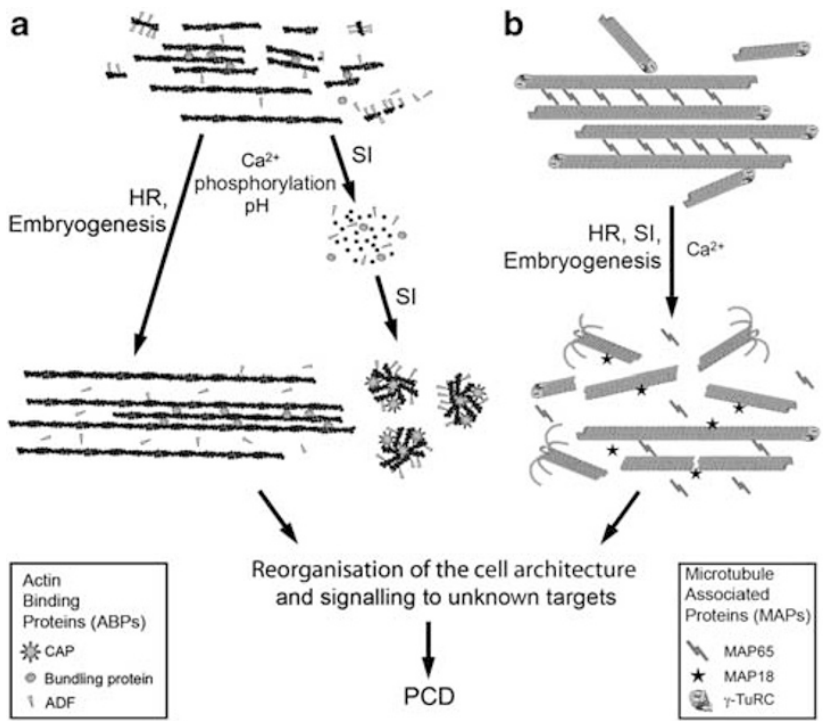

Figure 3 Regulation of cytoskeletal changes during PCD. (a) Regulation of F-actin dynamics during PCD in plants. In normal cells (upper part) ADF severs existing filaments and so promotes their disassembly, and provides new ends for polymerisation of filaments. Bundling proteins (e.g. fimbrin and villin) bundle F-actin. PCD induction (lower part) increases cytoplasmic concentration of $\mathrm{Ca}^{2+}$, upregulates protein kinase activity and reduces $\mathrm{pH}$. This can lead to at least two different scenarios. The first involves inhibition of ADF-severing activity, allowing formation of F-actin cables with the aid of bundling proteins. This has been observed in HR-PCD and embryo suspensor PCD. In the SI response, $\mathrm{Ca}^{2+}$ increases are likely to stimulate activation of a profilin and a gelsolin-like protein, leading to $\mathrm{F}$-actin depolymerisation. This is followed by de novo formation of highly stable F-actin foci associated with adenylate CAP and ADF. (b) Regulation of microtubule dynamics following PCD induction. In differentiated plant cells (upper part) microtubules are nucleated by $\gamma$-tubulin ring complexes ( $\gamma$-TuRC) in the cytoplasm or on the walls of existing microtubules (Hamada ${ }^{4}$ ). After nucleation microtubules can be bundled by MAP65. Initiation of PCD (lower part) and consequent changes in intracellular pH, mitogen-activated protein kinase (MAPK) activity, $\mathrm{Ca}^{2+}$ and NO concentration, can result in a reduction of microtubule nucleation and bundling, and upregulation of microtubule severing proteins like MAP18 and a reduction of the density and depolymerisation of cortical microtubules observed during HR and embryo suspensor differentiation. MAPs responsible for SI-induced microtubule depolymerisation have not been identified as yet

early differentiation in embryo suspensor, microtubules are decorated with MAP65. ${ }^{12}$ The onset of PCD is accompanied by a reduction in MAP65 gene transcription ${ }^{16}$ and the dissociation of MAP65 from microtubules. ${ }^{12}$ This is followed by fragmentation and depolymerisation of the microtubule network (Figure 3b). Transcription of a microtubule severing protein MAP18 increases upon activation of senescence, implicating it in driving disassembly of microtubules in this system. ${ }^{16}$ It is unknown whether some MAPs remain associated with fragmented microtubules to facilitate dismantling of the array or whether this is a consequence of, for example altered $\mathrm{pH}$ and cytosolic free $\mathrm{Ca}^{2+}\left(\left[\mathrm{Ca}^{2+}\right]_{\mathrm{cyt}}\right)$, which can inhibit tubulin polymerisation. The depolymerisation of microtubules during PCD in these model systems therefore appears to be controlled by a reduction of nucleation, inhibition of bundling, and increase in severing of microtubules. Thus, we are beginning to make the first steps in identifying proteins that may regulate the microtubule alterations during PCD in plants. However, to date there is no 
experimental evidence identifying MAPs involved in HR- and SI-PCD.

\section{Conclusions}

Information from several model systems provide compelling evidence that alterations in actin and tubulin polymerisation status is a key mechanism to mediate PCD in plant (this review) as well as in animal cells and yeast. ${ }^{46}$ There appears to be a consistent theme of $\mathrm{F}$-actin reorganisation achieved by alterations in dynamics mediating a decision-making role in regulating $\mathrm{PCD}$ in plant cells. This may involve cross-talk with microtubules. Data sometimes appears contradictory and this may reflect the fact that relatively few studies have examined this phenomenon, as well perhaps reflecting the heterogeneity of the signalling pathways in each system. Much remains to be learnt about the basis of how this is achieved. The cytoskeleton might serve as a dynamic and integrated feedback mechanism for 'health' status, as PCD signals major alterations to the polymeric status of actin and tubulin. At the same time it may also coordinate cytoplasm dismantling as PCD progresses. Tian et al. ${ }^{52}$ raised an interesting possibility that alterations to the plant actin cytoskeleton might signal directly to PCD. This was based on findings by $\mathrm{HaO}$ and August ${ }^{65}$ that showed that actin depolymerisation could activate signalling, enhance transcription factor activation, and prolong $\mathrm{Ca}^{2+}$ release in lymphocytes. An exciting possibility is that a similar scenario may operate in plant cells. Thus, an important area for future research, in addition to consolidating information about these alterations during PCD, is investigating how they are achieved: which signalling components are involved and which interacting proteins mediate these crucial life-or-death decisions.

\section{Conflict of Interest}

The authors declare no conflict of interest.

Acknowledgements. Work in the lab of VE F-T. is funded by the Biotechnology and Biological Sciences Research Council (B.B.S.R.C.).

1. Smertenko AP, Deeks MJ, Hussey PJ. Strategies of actin reorganisation in plant cells J Cell Sci 2010; 123: 3019-3029.

2. Staiger CJ, Poulter NS, Henty JL, Franklin-Tong VE, Blanchoin L. Regulation of actin dynamics by actin-binding proteins in pollen. J Exp Bot 2010; 61: 1969-1986.

3. Shaw SL, Kamyar R, Ehrhardt DW. Sustained microtubule treadmilling in Arabidopsis cortical arrays. Science 2003; 300: 1715-1718

4. Hamada T. Microtubule-associated proteins in higher plants. J Plant Res 2007; 120: 79-98.

5. Sedbrook JC. MAPs in plant cells: delineating microtubule growth dynamics and organization. Cur Opin Plant Biol 2004; 7: 632-640.

6. Staiger CJ (ed.). Actin: A dynamic framework for multiple plant cell functions. Kluwer Academic Publishers: Dordrecht, The Netherlands, 2000.

7. Fukuda $\mathrm{H}$, Kobayashi $\mathrm{H}$. Dynamic organization of the cytoskeleton during trachearyelement differentiation. Dev Growth Differ 1989; 31: 9-16.

8. Oda Y, Hasezawa S. Cytoskeletal organization during xylem cell differentiation. J Plant Res 2006; 119: 167-177.

9. Kobayashi H, Fukuda H, Shibaoka H. Interrelation between the spatial disposition of actinfilaments and microtubules during the differentiation of tracheary elements in cultured zinnia cells. Protoplasma 1988; 143: 29-37.

10. Oda Y, Mimura T, Hasezawa S. Regulation of secondary cell wall development by cortical microtubules during tracheary element differentiation in Arabidopsis cell suspensions. Plant Phys 2005; 137: 1027-1036.
11. Bozhkov PV, Filonova LG, Suarez MF. Programmed cell death in plant embryogenesis. Curr Topics Plant Dev 2005; 67: 135-179.

12. Smertenko AP, Bozhkov V, Filonova LH, von Arnold S, Hussey PJ. Re-organisation of the cytoskeleton during developmental programmed cell death in Picea abies embryos. Plant $J$ 2003; 33: 813-824.

13. Schwarzerova K, Vondrakova Z, Fischer L, Borikova P, Bellinvia E, Eliasova K et al. The role of actin isoforms in somatic embryogenesis in Norway spruce. BMC Plant Biol 2010; 10: 89.

14. Swidzinski JA, Sweetlove LJ, Leaver CJ. A custom microarray analysis of gene expression during programmed cell death in Arabidopsis thaliana. Plant $J$ 2002; 30: 431-446.

15. Bai SY, Willard B, Chapin LJ, Kinter MT, Francis DM, Stead AD et al. Proteomic analysis of pollination-induced corolla senescence in petunia. J Exp Bot 2010; 61: 1089-1109.

16. Keech $O$, Pesquet E, Gutierrez L, Ahad A, Bellini C, Smith $S$ et al. Leaf senescence is accompanied by an early disruption of the microtubule network in Arabidopsis. Plant Phys 2010; 154: 1710-1720.

17. McClure B, Franklin-Tong V. Gametophytic self-incompatibility: understanding the cellular mechanisms involved in 'self' pollen tube inhibition. Planta 2006; 224: 233-245.

18. Foote HCC, Ride JP, Franklin-Tong VE, Walker EA, Lawrence MJ, Franklin FCH. Cloning and expression of a distinctive class of self- incompatibility (S) gene from Papaver rhoeas L. Proc Nat Acad Scie USA 1994; 91: 2265-2269.

19. Wheeler MJ, de Graaf BHJ, Hadjiosif N, Perry RM, Poulter NS, Osman K et al. Identification of the pollen self-incompatibility determinant in Papaver rhoeas. Nature 2009; 459: 992-995

20. Thomas SG, Franklin-Tong VE. Self-incompatibility triggers programmed cell death in Papaver pollen. Nature 2004; 429: 305-309.

21. Bosch M, Franklin-Tong VE. Temporal and spatial activation of caspase-like enzymes induced by self-incompatibility in Papaver pollen. Proc Natl Acad Sci USA 2007; 104: 18327-18332.

22. Bosch M, Franklin-Tong VE. Self-incompatibility in Papaver. signalling to trigger PCD in incompatible pollen. J Exp Bot 2008; 59: 481-490.

23. Wilkins KA, Bancroft J, Bosch M, Ings J, Smirnoff N, Franklin-Tong VE et al. ROS and NO are involved in the self-incompatibility response of Papaver. Plant Physiology, doi 10.1104/ pp.110.167510.

24. Li S, Samaj J, Franklin-Tong VE. A mitogen-activated protein kinase signals to programmed cell death induced by self-incompatibility inPapaver pollen. Plant Physio 2007; 145: 236-245.

25. Geitmann A, Franklin-Tong VE, Emons AMC. The self-incompatibility response in Papaver rhoeas pollen causes early and striking alterations to organelles. Cell Death Differ 2004; 11: 812-822.

26. Jordan ND, Franklin FCH, Franklin-Tong VE. Evidence for DNA fragmentation triggered in the self- incompatibility response in pollen of Papaver rhoeas. Plant J 2000; 23: 471-479.

27. Geitmann AB, Snowman BN, Emons AMC, Franklin-Tong VE. Alterations in the actin cytoskeleton of pollen tubes are induced by the self-incompatibility reaction in Papaver rhoeas. Plant Cell 2000; 12: 1239-1252.

28. Snowman BN, Kovar DR, Shevchenko G, Franklin-Tong VE, Staiger CJ. Signal-mediated depolymerization of actin in pollen during the self-incompatibility response. Plant Cell 2002; 14: 2613-2626.

29. Poulter NS, Staiger CJ, Rappoport JZ, Franklin-Tong VE. Actin-binding proteins implicated in formation of the punctate actin foci stimulated by the self-incompatibility response in Papaver. Plant Physiol 2010; 152: 1274-1283.

30. Poulter NS, Bosch M, Franklin-Tong VE. Proteins implicated in mediating SelfIncompatibility-induced alterations to the actin cytoskeleton of Papaver pollen. Ann Bot 2011; doi: 10.1093/aob/mcr022.

31. Poulter NS, Vatovec S, Franklin-Tong VE. Microtubules are a target for self-incompatibility signaling in Papaver pollen. Plant Physiol 2008; 146: 1358-1367.

32. Wang $\mathrm{CL}, \mathrm{Xu} \mathrm{GH}$, Jiang XT, Chen G, Wu J, Wu HQ et al. S-RNase triggers mitochondrial alteration and DNA degradation in the incompatible pollen tube of Pyrus pyrifolia in vitro. Plant J 2009; 57: 220-229.

33. Liu ZQ, Xu GH, Zhang SL. Pyrus pyrifolia stylar S-RNase induces alterations in the actin cytoskeleton in self-pollen and tubes in vitro. Protoplasma 2007; 232: 61-67.

34. Wang CL, Wu J, Xu GH, Gao YB, Chen G, Wu JY et al. S-RNase disrupts tip-localized reactive oxygen species and induces nuclear DNA degradation in incompatible pollen tubes of Pyrus pyrifolia. J Cell Sci 2010; 123: 4301-4309.

35. Greenberg JT. Programmed cell death: a way of life for plants. Proc Nat Acad Sci USA 1996; 93: 12094-12097.

36. Lam E, Kato N, Lawton M. Programmed cell death, mitochondria and the plant hypersensitive response. Nature 2001; 411: 848-853.

37. Greenberg JT, Yao N. The role and regulation of programmed cell death in plant-pathogen interactions. Cell Microbiol 2004; 6: 201-211.

38. Lam E. Controlled cell death, plant survival and development. Nat Rev Mol Cell Biol 2004; 5: $305-315$

39. Skalamera D, Heath MC. Changes in the cytoskeleton accompanying infection-induced nuclear movements and the hypersensitive response in plant cells invaded by rust fungi. Plant J 1998; 16: 191-200.

40. Takemoto D, Jones DA, Hardham AR. GFP-tagging of cell components reveals the dynamics of subcellular re-organization in response to infection of Arabidopsis by oomycete pathogens. Plant J 2003; 33: 775-792. 
41. Higaki T, Goh T, Hayashi T, Kutsuna N, Kadota Y, Hasezawa S et al. Elicitor-induced cytoskeletal rearrangement relates to vacuolar dynamics and execution of cell death: in vivo imaging of hypersensitive cell death in tobacco BY-2 cells. Plant Cell Phys 2007; 48: 1414-1425

42. Kobayashi I, Kobayashi Y, Hardham AR. Dynamic reorganization of microtubules and microfilaments in flax cells during the resistance response to flax rust infection. Planta 1994; 195: 237-247.

43. Takemoto D, Jones DA, Hardham AR. Re-organization of the cytoskeleton and endoplasmic reticulum in the Arabidopsis pen1-1 mutant inoculated with the nonadapted powdery mildew pathogen, Blumeria graminis f. sp hordei. Mol Plant Pathol 2006; 7: $553-563$.

44. Cahill D, Rookes J, Michalczyk A, McDonald K, Drake A. Microtubule dynamics in compatible and incompatible interactions of soybean hypocotyl cells with Phytophthora sojae. Plant Pathol 2002; 51: 629-640.

45. Binet MN, Humbert C, Lecourieux D, Vantard M, Pugin A. Disruption of microtubular cytoskeleton induced by cryptogein, an elicitor of hypersensitive response in tobacco cells. Plant Physiol 2001; 125: 564-572.

46. Franklin-Tong VE, Gourlay CW. A role for actin in regulating apoptosis/programmed cell death: evidence spanning yeast, plants and animals. Biochem J 2008; 413: 389-404.

47. Thomas SG, Huang SJ, Li ST, Staiger CJ, Franklin-Tong VE. Actin depolymerization is sufficient to induce programmed cell death in self-incompatible pollen. J Cell Biol 2006; 174: $221-229$

48. Miklis M, Consonni C, Bhat RA, Lipka V, Schulze-Lefert P, Panstruga R. Barley $\mathrm{mLO}$ Modulates actin-dependent and actin-independent antifungal defense pathways at the cell periphery. Plant Physiol 2007; 144: 1132-1143.

49. Tomiyama K, Sato K, Doke N. Effect of cytochalasin b and colchicine on hypersensitive death of potato cells infected by incompatible race of Phytophthora-infestans. Ann Phytopathol Soc Japan 1982; 48: 228-230.

50. Takemoto D, Maeda H, Yoshioka H, Doke N, Kawakita K. Effect of cytochalasin D on defence responses of potato tuber discs treated with hyphal wall components of Phytophthora infestans. Plant Sci 1999; 141: 219-226.

51. Kobayashi $Y$, Kobayashi I. Depolymerization of the actin cytoskeleton induces defense responses in tobacco plants. J Gen Plant Pathol 2007; 73: 360-364.

52. Tian M, Chaudhry F, Ruzicka DR, Meagher RB, Staiger CJ, Day B. Arabidopsis actindepolymerizing factor AtADF4 mediates defense signal transduction triggered by the Pseudomonas syringae effector AvrPphB. Plant Physiol 2009; 150: 815-824.

53. Schmelzer E. Cell polarization, a crucial process in fungal defence. Trends Plant Sci 2002; 7: $411-415$.
54. Maciver SK, Hussey PJ. The ADF/cofilin family: actin-remodeling proteins. Genome Biol 2002; 3: 3007.1-3007.12

55. Smertenko AP, Jiang CJ, Simmons NJ, Weeds AG, Davies DR, Hussey PJ. Ser6 in the maize actin-depolymerizing factor, ZmADF3, is phosphorylated by a calcium-stimulated protein kinase and is essential for the control of functional activity. Plant $J$ 1998; 14 187-194.

56. Huang S, Blanchoin L, Chaudhry F, Franklin-Tong VE, Staiger CJ. A gelsolin-like protein from Papaver rhoeas pollen (PrABP80) stimulates calcium-regulated severing and depolymerization of actin filaments. J Biol Chem 2004; 279: 23364-23375.

57. Lila T, Drubin DG. Evidence for physical and functional interactions among two Saccharomyces cerevisiae SH3 domain proteins, an adenylyl cyclase-associated protein and the actin cytoskeleton. Mol Biol Cell 1997; 8: 367-385.

58. Sagot I, Pinson B, Salin B, Daignan-Fornier B. Actin bodies in yeast quiescent cells: an immediately available actin reserve? Mol Biol Cell 2006; 17: 4645-4655.

59. Gourlay CW, Carpp LN, Timpson P, Winder SJ, Ayscough KR. A role for the actin cytoskeleton in cell death and aging in yeast. J Cell Biol 2004; 164: 803-809.

60. McLusky SR, Bennett MH, Beale MH, Lewis MJ, Gaskin P, Mansfield JW. Cell wall alterations and localized accumulation of feruloyl- $3^{\prime}$-methoxytyramine in onion epidermis at sites of attempted penetration by Botrytisallii are associated with actin polarisation, peroxidase activity and suppression of flavonoid biosynthesis. Plant $J$ 1999; 17: $523-534$.

61. Shimada C, Lipka V, O'Connell R, Okuno T, Schulze-Lefert P, Takano Y. Nonhost resistance in Arabidopsis-Colletotrichum interactions acts at the cell periphery and requires actin filament function. Mol Plant Microbe Inter 2006; 19: 270-279.

62. Shan XC, Goodwin PH. Reorganization of filamentous actin in Nicotiana benthamiana lea epidermal cells inoculated with Colletotrichum destructivum and Colletotrichum graminicola. Int J Plant Sci 2005; 166: 31-39.

63. Barton DA, Vantard M, Overall RL. Analysis of cortical arrays from Tradescantia virginiana at high resolution reveals discrete microtubule subpopulations and demonstrates that confocal images of arrays can be misleading. Plant Cell 2008; 20: 982-994.

64. Chan J, Jensen CG, Jensen LC, Bush M, Lloyd CW. The 65-kDa carrot microtubuleassociated protein forms regularly arranged filamentous cross-bridges between microtubules. Proc Natl Acad Sci USA 1999; 96: 14931-14936.

65. Hao S, August A. Actin depolymerization transduces the strength of B-cell receptor stimulation. Mol Biol Cell 2005; 16: 2275-2284.

66. Shi F-M, Yao L-L, Pei B-L, Zhou Q, Li X-L, Li Y et al. Cortical microtubule as a sensor and target of nitric oxide signal during the defence responses to Verticillium dahliae toxins in Arabidopsis. Plant Cell Evir 2009; 32: 428-438. 